\title{
Aggregation-induced symmetry breaking from achiral systems
}

\author{
Haoke Zhang ${ }^{1,2}$, Ryan T. K. Kwok ${ }^{1,2}$, Jacky W. Y. Lam ${ }^{1,2}$, and Ben Zhong Tang ${ }^{1,2,3,4 *}$
}

1. Department of Chemistry, Hong Kong Branch of Chinese National Engineering Research Center for Tissue Restoration and Reconstruction and Institute for Advanced Study, The Hong Kong University of Science and Technology Clear Water Bay, Kowloon, Hong Kong 999077, China.

2. HKUST-Shenzhen Research Institute, No. 9 Yuexing 1st Rd, South Area, Hi-tech Park, Nanshan, Shenzhen 518057, China.

3. Center for Aggregation-Induced Emission, State Key Laboratory of Luminescent Materials and Devices, SCUT-HKUST Joint Research Institute, South China University of Technology, Tianhe Qu, Guangzhou 510640, China.

4. AIE Institute, Guangzhou Development District, Huangpu, Guangzhou 510530, China Correspondence and requests for materials should be addressed to B.Z.T. (email: tangbenz@ust.hk)

\begin{abstract}
Debates on the source of chirality have lasted for several centuries due to its significance for the exploration of the origin of life. It is still a thorny scientific problem that how the first chiral molecule is generated without any chiral factors. In this work, an interesting phenomenon, aggregation-induced symmetry breaking (AISB), is observed in achiral diphenylethylene and $p$-terphenyl derivatives. For compound (E)-1,2-Bis(2,4,5trimethylphenyl)ethene (2,4,5-TMe-DPE), there is no chiroptical signal both in its solution and crystal state. However, unexpected chiroptical signals, circular dichroism (CD) and circularly polarized luminescence (CPL), are observed in their aggregate state and the emission dissymmetry factor is as high as 0.015 at $70 \%$ water fraction. Meantime, time-dependent CD and CPL effect are also investigated which suggests that AISB involves both dynamic and kinetic processes. Calculation is further carried out to prove the existence of two enantiomers in diphenylethylene and $p$-terphenyl derivatives. Finally, we propose that the symmetry breaking in aggregate state might be caused by the difference of resolution rate to two enantiomers.
\end{abstract}




\section{Introduction}

Chirality showed a close relationship with the life in the earth, which has been widely investigated in many different areas, such as chemistry, biology, physics and mathematics. A large number of chiral molecules are the elementary components of life. For example, most of the transcriptional active DNA is right-handed, all the amino acids in the living bodies are levorotatory but the sugars are dextrorotatory. Meanwhile, most of the drugs show chiral structure. Clinical results indicated that, in most cases, only one of the enantiomers could treat the diseases but the other one always plays a negative role. Our body is a small factory that efficiently and constantly produces chiral molecules. However, among all these processes, the generation of chiral compounds is highly dependent on the introduction of chiral factors. For instance, the dominance of $L$-amino acid in our body is originated from the chiral biocatalysis of enzymes.

However, the emergence of the first chiral structure on earth is still full of mystique. Many different viewpoints have been put forwarded in the past several decades. For example, some researchers have set up experiments to demonstrate that the circularly polarized light from sunlight could facilitate the chiral synthesis. Other scientists proposed that the chiral catalysts came from the volcanic eruption. Among all these hypotheses, although some of them look like reasonable, the lack of direct evidence makes them unconvincing.

In the course of our research, we discovered the relationship between molecular aggregation and chiral separation ${ }^{1-5}$. In this work, we have synthesized a series of diphenylethylene (DPE) and p-terphenyl derivatives. These molecules showed no chiroptical signal in their good solvent. However, unexpected strong circular dichroism (CD) and circularly polarized luminescence (CPL) signals were observed once the aggregates were formed by adding their poor solvent. Meanwhile, we found that the generation of chiral signal in the aggregate state was a dynamic process. For example, $(E)$-1,2-Bis(2,4,5-trimethylphenyl)ethene (2,4,5-TMe-DPE) initially had no chiroptical signal when the aggregates were immediately formed. However, with the time going on, the CD signal from $(R)-2,4,5-\mathrm{TMe}-\mathrm{DPE}$ appeared first and the intensity was gradually enhanced. At a critical point, the intensity began to decrease and the signal from $(S)$-2,4,5-TMe-DPE was dominated. The observed phenomenon in this project is quite interesting and could make a great contribution to the explanation of the origin of chirality from achiral systems. 


\section{Results}

Diphenylethylene (DPE) and p-terphenyl derivatives in this project were synthesized in our previously published works ${ }^{6,7}$. All the molecules were characterized and confirmed by NMR and mass spectroscopy. Suitable crystals were grown from their solutions and characterized crystallographically. As shown in Figure 1, the resultant 2,4,5-TMe-DPE showed achiral structure in its crystal packing that the two phenyl rings were parallel to each other. The twist angle between the benzene ring and the plane of middle double bond was $27^{\circ}$. Meanwhile, no strong $\pi-\pi$ interaction was observed in this system except the intermolecular through-space hyperconjugation.

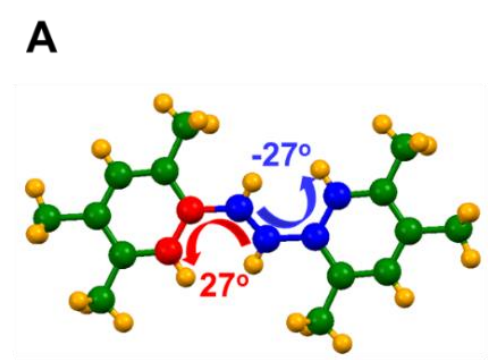

C

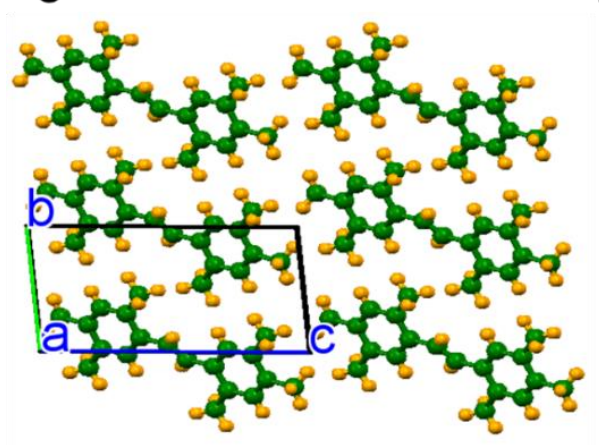

B

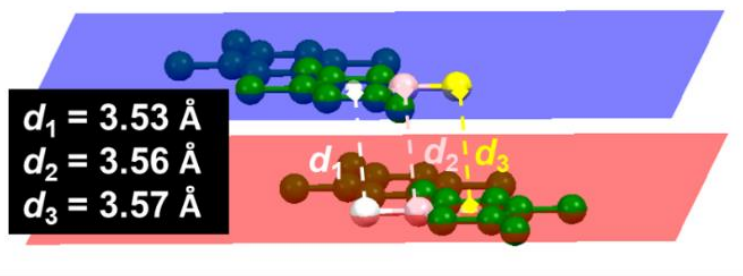

D

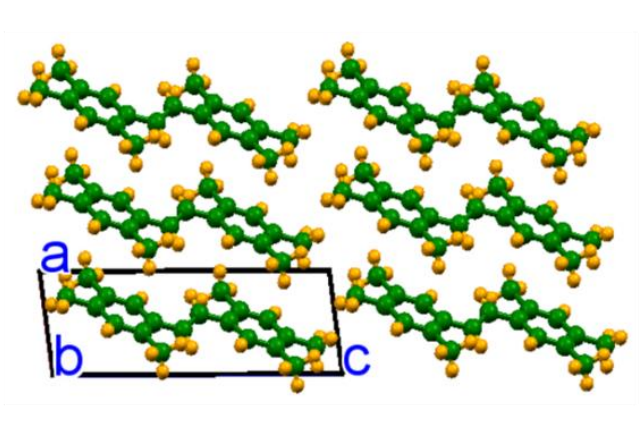

Figure 1. A) Crystal structure, B), C) and D) packing structures of 2,4,5-TMe-DPE.

To verify the achiral structure in crystalline state, solid-state CD spectra were measured for 2,4,5-TMe-DPE. We have doped its crystal into potassium bromide with weight ratio 1/200 and the transparent $\mathrm{KBr}$ pellet was prepared ${ }^{8}$. As expected, no $\mathrm{CD}$ signal has been obtained. Then the crystal was dissolved in its good solvent, tetrahydrofuran (THF), and a $100 \mu \mathrm{M}$ solution was prepared. The solution CD showed that there was no obvious signal in the pure THF solution. Then, its poor solvent of water was gradually added to form the aggregates and the mixtures were still CD silent when the water fraction $\left(f_{\mathrm{w}}\right)$ was lower than $60 \%$ (Figure 2$)^{9}$. However, a strong CD signal was observed when $f_{\mathrm{w}}$ reached $70 \%$. Figure 2 indicated that there were three peaks in their CD spectra, 
two positive peaks at 211 and $270 \mathrm{~nm}$ and a negative peak at $245 \mathrm{~nm}$. With the increase of water fraction, the molar ellipticity of these two positive peaks was decreased but an increase was exhibited in the negative peak. All the samples were measured after standing for $2 \mathrm{~h}$. Meanwhile, the observed phenomenon was repeatable under the same condition. It was unexpected and mysterious how such an achiral molecule could generate strong CD signal. At the same time, the excited-state chiroptical properties were also investigated by CPL.

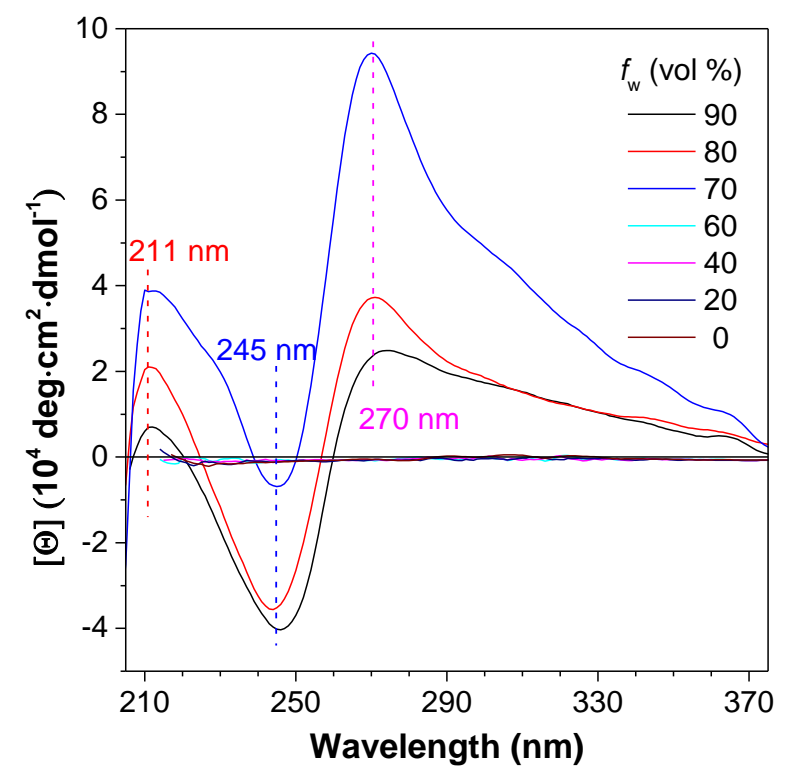

Figure 2. Circular dichroism spectra of 2,4,5-TMe-DPE in THF/water mixtures (100 $\mu \mathrm{M})$ with different water fractions $\left(f_{\mathrm{w}}\right)$.

Emission dissymmetry factor $\left(g_{\text {em }}\right)$ is an important parameter to evaluate the performance of CPL, which is defined as $2\left(I_{\mathrm{L}}-I_{\mathrm{R}}\right) /\left(I_{\mathrm{L}}+I_{\mathrm{R}}\right)$ and $I_{\mathrm{L}}$ and $I_{\mathrm{R}}$ represented the intensity of left- and right-hand light, respectively. Generally, for the chiral lanthanide complexes, the $g_{\text {em }}$ could achieve to $0.1-0.5$ or even as high as $1.4^{10}$. However, for the chiral organic molecules, the value always located in the levels of $10^{-5}-10^{-2}$. As shown in Figure 3, no CPL signal was achieved at $f_{\mathrm{w}}=0 \%$. However, a strong CPL signal was observed once the $f_{\mathrm{w}}$ was increased to $70 \%$. The strongest CPL signal located at $70 \%$ and the intensity showed a slight decrease from $70 \%$ to $90 \%$ which was similar to the CD spectra. It is noteworthy that the maximum dissymmetry factor at $70 \%$ reached up to 0.015. As an untreated organic sample, this value almost arrived at a limit. Moreover, the CPL signal was obtained in the aggregate state, which had a promising prospect in the application. 

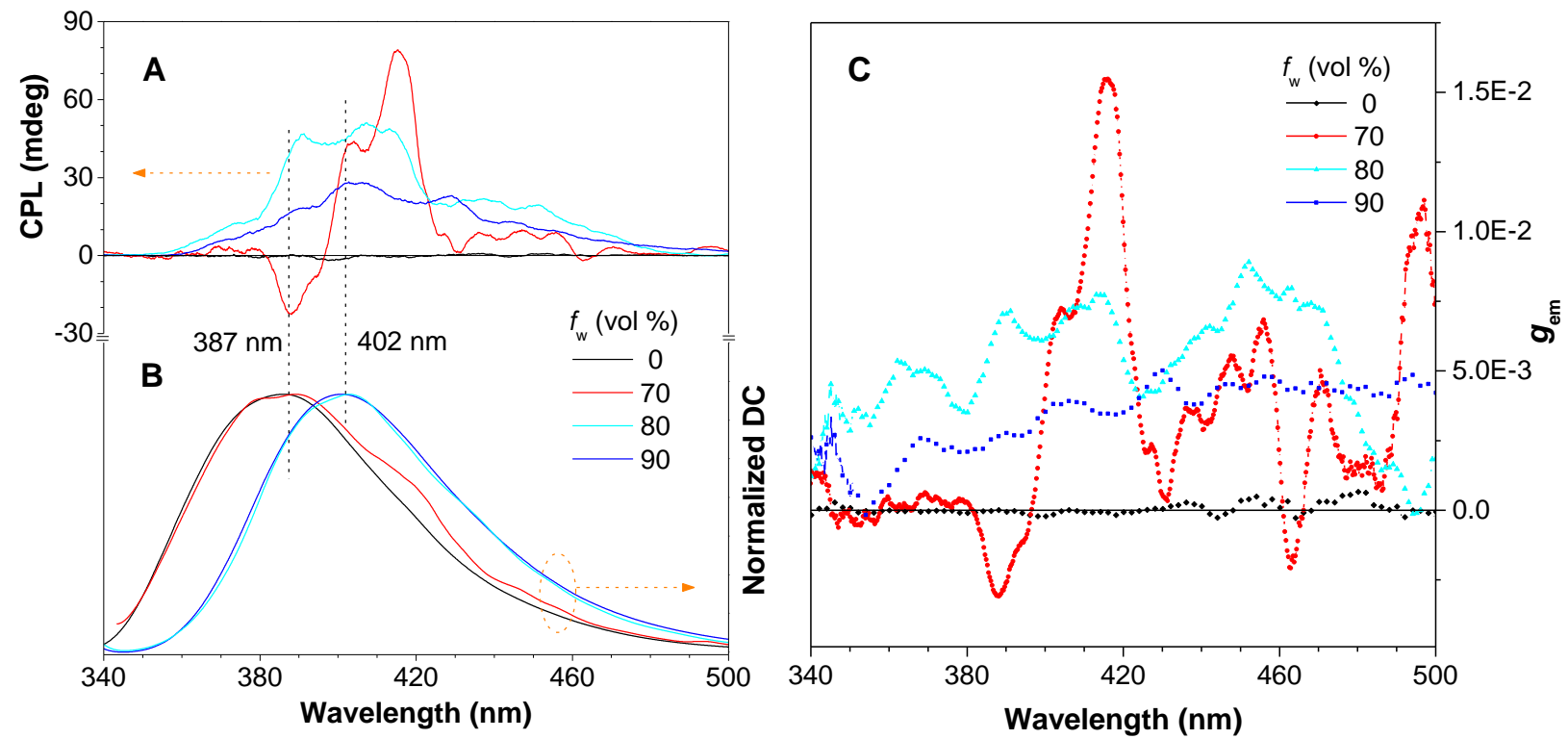

Figure 3. A) Circularly polarized luminescence (CPL), B) total luminescence and C) CPL dissymmetry factor $\left(g_{\mathrm{em}}\right)$ of 2,4,5-TMe-DPE in THF/water mixtures with different water fractions $\left(f_{\mathrm{w}}\right)$. Concentration: $100 \mu \mathrm{M}$.

To clarify the source of aggregate chirality. Time-dependent CD spectra were recorded for 2,4,5-TMe-DPE in its THF/water mixture at $f_{\mathrm{w}}=60,70,80$ and 90\%, the sample was scanned at different standing time (Figure 4). At $f_{\mathrm{w}}=60 \%$, no CD signal was observed even at 550 min (Figure $4 \mathrm{~A})$. For $f_{\mathrm{w}}=70$ and $80 \%, \mathrm{CD}$ signal is also silent at the beginning but a negative signal at $272 \mathrm{~nm}$ was gradually increased with the time going on. At a critical point, the CD intensity began to decrease and the positive signal was dominant at last (Figure 4B and C). Sample of 90\% showed different behavior to 70 and $80 \%$. Although CD signal was still silent at the beginning, the positive signal was obtained directly with time going on and the intensity was gradually increased (Figure 4D). Figure 4E-F has demonstrated the change of molar ellipticity $([\Theta])$ at $272 \mathrm{~nm}$ with different standing time. For example, for $70 \%$ sample, the negative $[\Theta]$ spent 20 minutes in arriving at the maximum. This period was shortened to 10 minutes at $f_{\mathrm{w}}=80 \%$. As mentioned above, no negative $[\Theta]$ was observed at $f_{\mathrm{w}}=90 \%$. 

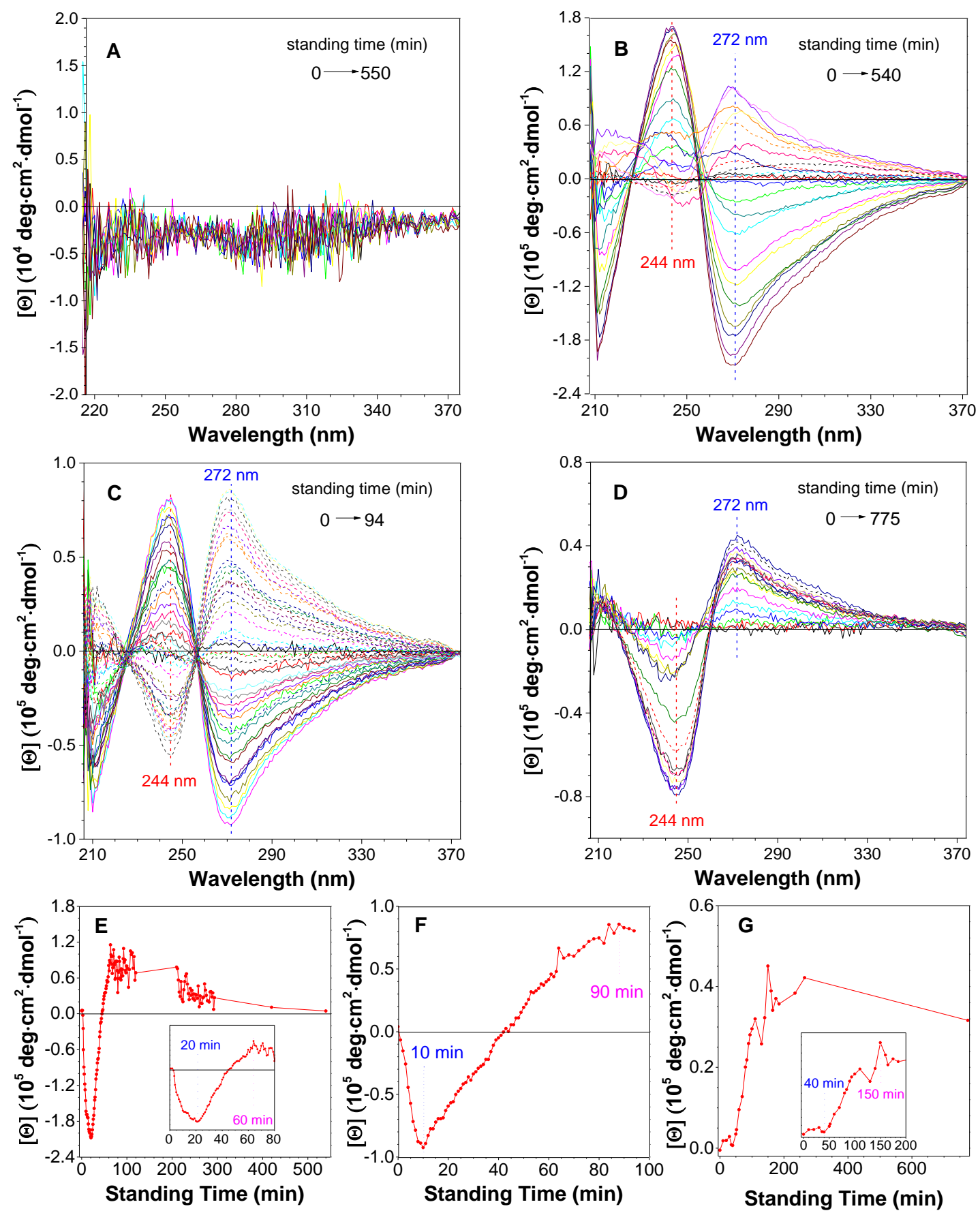

Figure 4. (A-D) CD spectra of 2,4,5-TMe-DPE in THF/water mixtures $(50 \mu \mathrm{M})$ with different water fractions $\left.\left.\left.\left(f_{\mathrm{w}}\right), \mathrm{A}\right) 60 \%, \mathrm{~B}\right) 70 \%, \mathrm{C}\right) 80 \%$ and D) $90 \%$ under different standing time. (E-G) Plots of CD value at $272 \mathrm{~nm}$ versus different standing time, $f_{\mathrm{w}}=$ E) $70 \%$, F) $80 \%$ and G) $90 \%$.

The dynamic change of CPL spectra at $f_{\mathrm{w}}=70-90 \%$ was also investigated (Figure S1-3). The fresh samples showed no CPL signal. With the increase of standing time, a positive CPL signal around $410 \mathrm{~nm}$ was induced and its intensity was gradually enhanced. By comparison to the time- 
dependent CD spectra, the ground and excited-state chiroptical properties showed a big difference. There was no negative signal within the whole process. The above-mentioned time-dependent CD and CPL results indicated that the kinetic symmetry breaking indeed existed in the process of aggregation. Time-dependent PL spectra were also recorded which further proved the existence of molecular motion in the aggregate state (Figure S4-7).
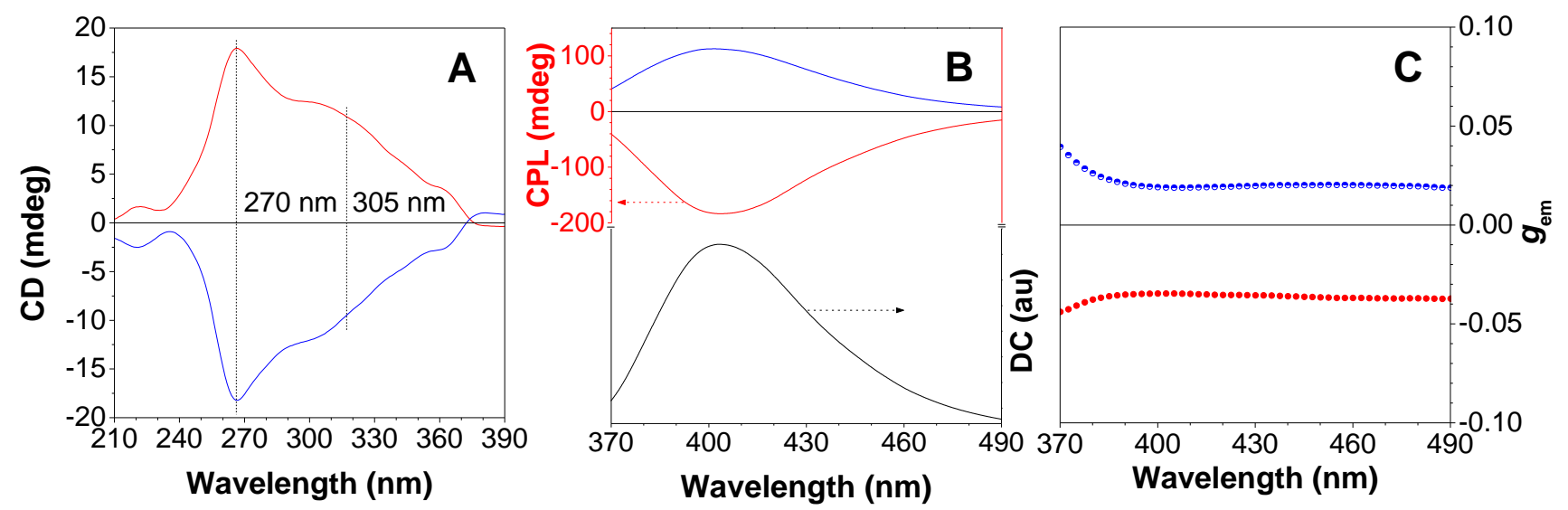

Figure 5. The chiroptical properties of 2,4,5-TMe-DPE deposited on quartz substrate. A) CD spectra B) $\mathrm{CPL}$ and total luminescence, C) CPL dissymmetry factor $\left(g_{\mathrm{em}}\right)$.

Strong CD and CPL signals have been detected in the aggregates of 2,4,5-TMe-DPE. However, its single crystal showed no chiroptical signal which was ascribed to the intramolecular parallel structure. So whether the film-state sample possessed chiroptical properties or not? Then, a thin film was prepared by spin-coating its DCE solution on the quartz substrate. Firstly, the CD spectra were measured in different areas of the film. Surprisingly, both negative and positive signals were obtained in the same film (Figure 5A). Ten pieces of films were prepared by the same methods and more than 100 spectra were recorded which indicates that the probability of negative and positive signal are both around $50 \%$. This phenomenon was not hard to understand and explain as lots of literature has already reported ${ }^{11,12}$. The process of evaporation induced the generation of self-assembly and molecules with the same chirality packed together, which was also a process of chiral separation $^{13}$. The CPL measurement almost exhibited the same phenomenon (Figure 5B and C). Both negative and positive signals were observed and the maximum $g_{\text {em }}$ reached up to -0.04 .

The same phenomenon was also observed in 2,4,5-TPh-TM in which the middle double bond was replaced by benzene. As shown in Figure 6A, weak positive CD signal at $250 \mathrm{~nm}$ was 
unexpectedly observed even at pure THF, then the intensity showed a slight increasing and the wavelength gradually shifted to $265 \mathrm{~nm}$ from $f_{\mathrm{w}}=0 \%$ to $60 \%$. Interestingly, an accidental spectrum with two negative peaks at 245 and $298 \mathrm{~nm}$ was detected at $70 \%$. Then, these two peaks suddenly changed to positive when the water fraction was increased to $80 \%$ and $90 \%$. The samples used in Figure 6A have stood for 30 minutes after the fresh preparation. Then, another group of samples with 120 minutes standing time was also measured (Figure 6B). Unlike the mirror-image CD spectra of samples shown in Figure 6A, 70-90\% mixtures in this group showed similar CD spectra which had two positive peaks at 236 and $300 \mathrm{~nm}$. CPL spectra were also recorded for 2,4,5-TPhTM at aggregate state (Figure 6C and D). A positive peak was observed both at $80 \%$ and $90 \%$ but the $g_{\text {em }}$ value was smaller than 2,4,5-TMe-DPE, only 0.004 .
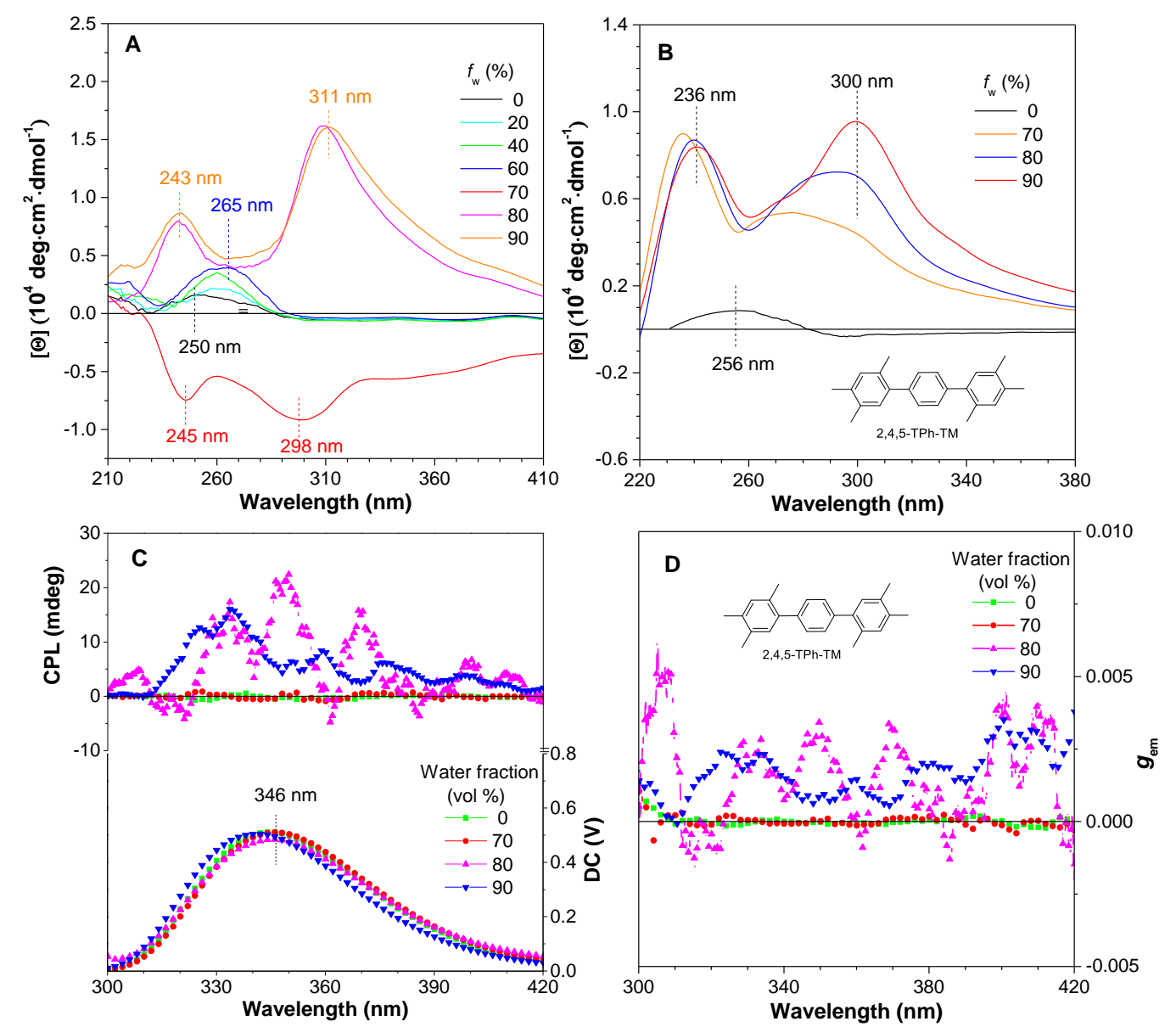

Figure 6. A-B) Circular dichroism spectra of 2,4,5-TPh-TM in THF/water mixtures (100 $\mu \mathrm{M})$ with different water fractions $\left(f_{\mathrm{w}}\right)$ at different standing time A) $30 \mathrm{~min}$ and B) $120 \mathrm{~min}$. C) Circularly polarized luminescence (CPL) and total luminescence and D) CPL dissymmetry factor $\left(g_{\mathrm{em}}\right)$ of 2,4,5-TPh-TM in $\mathrm{THF} /$ water mixtures with different water fractions $\left(f_{\mathrm{w}}\right)$. Concentration $=100 \mu \mathrm{M}$. 
In order to clarify the source of aggregation-induced symmetry breaking (AISB), microstructures of 2,4,5-TMe-DPE were investigated. Firstly, 2,4,5-TMe-DPE was dissolved in dichloroethane (DCE) and then the spin-coating was used to prepare the film on the glass slides. DCE/hexane mixture $(1 / 9, v / v)$ was also used to prepare the samples. Fluorescence microscopy results suggested that branch-like structures were both observed in pure DCE and DCE/hexane mixture with 90\% hexane fraction. The beautiful and regular structure showed bright blue fluorescence. The pictures captured under daylight seem like some helical structures were formed. However, as the scale of these structures were around several micrometers, so it was difficult to differentiate whether these helical structures were the real morphology or not. The electron microscopy results suggested that snowflake structure was observed but without clear helical structures (Figure S14). The morphological studies suggested that the AISB may not show a close relationship with the formation of regular and directional structures. Then, the theoretical calculation was further carried out to clarify the AISB at molecular level.

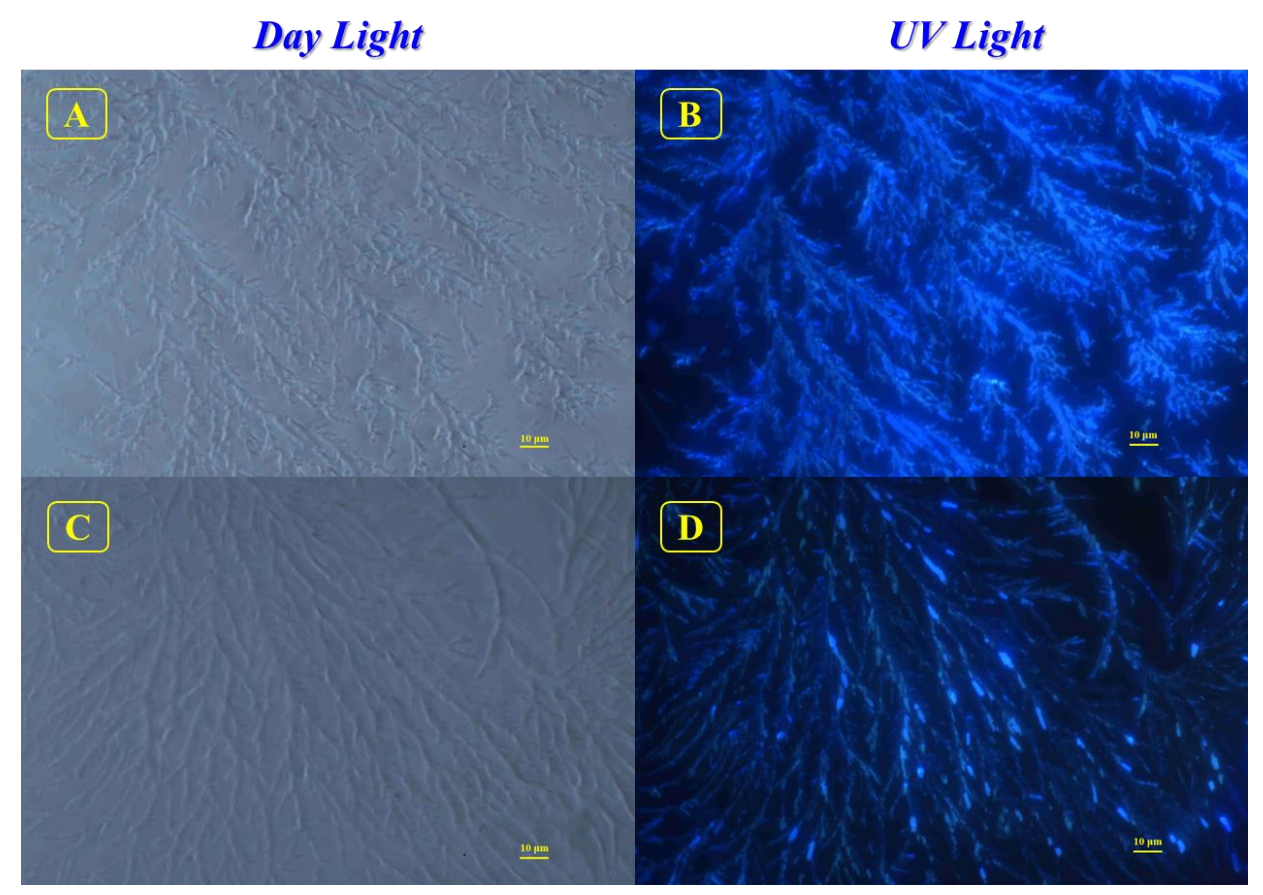

Figure 7. Fluorescence microscopy of the film of 2,4,5-TMe-DPE evaporated from the DCE/hexane mixture, hexane fraction $\left(f_{\mathrm{h}}\right)$ : A) and B) $0 \%$, C) and D) $90 \%$.

As mentioned above, two benzene rings in 2,4,5-TMe-DPE showed a parallel structure in single crystal and the dihedral angle between the benzene ring and the plane of double bond was $27.2^{\circ}$. After the structural optimization, the dihedral angle was decreased to $21.4^{\circ}$ but still with a parallel structure (Figure S15A). Then, the energy plot was calculated with the variation of dihedral angle $(\alpha=-\beta)$ (Figure S15B). As shown in Figure S15C, all these parallel structures 
showed no CD signal which explained why the CD signal has not been detected in the crystal and pure THF solution. When the value of $\alpha$ equals to $\beta, 2,4,5-\mathrm{TMe}-\mathrm{DPE}$ exhibited an intersectant conformation. The plot of single-point energy versus twist angle was also calculated and plotted. As shown in Figure $8 \mathrm{~A}$, the minimum energy was still located at $\alpha=\beta \approx \pm 20^{\circ}$. Simulation results showed that strong CD signal was obtained in intersectant structures (Figure 8B). Figure $8 \mathrm{C}$ indicated that positive signal was induced when $\alpha=\beta<0^{\circ}$ and vice versa. Meanwhile, the $|[\Theta]|$ was increased with the increase of $|\alpha|$. According to the conjugation theory, the more planar structure the narrower band gap and that was the reason why a blue shift was observed when the $|\alpha|$ was increased (Figure 8D). As the conformation shown in Figure S15 and 8A-D is not the optimized result, so it was hard to conclude which conformation was more stable, parallel or intersectant structure. In Figure $8 \mathrm{E}$, the $\alpha$ and $\beta$ were initially set as $-40^{\circ}$ and $20^{\circ}$, respectively, and the dihedral angle of $\alpha$ was gradually increased from $-40^{\circ}$ to $40^{\circ}$ with a $5^{\circ}$ interval. The final potential curve suggested that the most stable conformation was $\alpha=\beta=25^{\circ}$ which was an intersectant structure. Figure 8F exhibited all the CD spectra at different twist angle of $\alpha$. Interestingly, when the initial conformation was set as $\alpha=-40^{\circ}, \beta=-20^{\circ}$ and the dihedral angle of $\alpha$ was also gradually increased from $-40^{\circ}$ to $40^{\circ}$ with a $5^{\circ}$ interval, the most stable conformation was disclosed as $\alpha=\beta=-25^{\circ}$ (Figure S16). The results indicated that there was a barrier when the phenyl ring intended to flap from one side to the other. The above-mentioned calculation revealed the structures of two enantiomers in 2,4,5-TMe-DPE and the obtained CD signal at aggregate state $\left(f_{\mathrm{w}}=70-90 \%\right)$ belonged to the structure with $\alpha=\beta=-25^{\circ}$ which is the $(S)-2,4,5-\mathrm{TMe}-\mathrm{DPE}$ (Figure S17). 

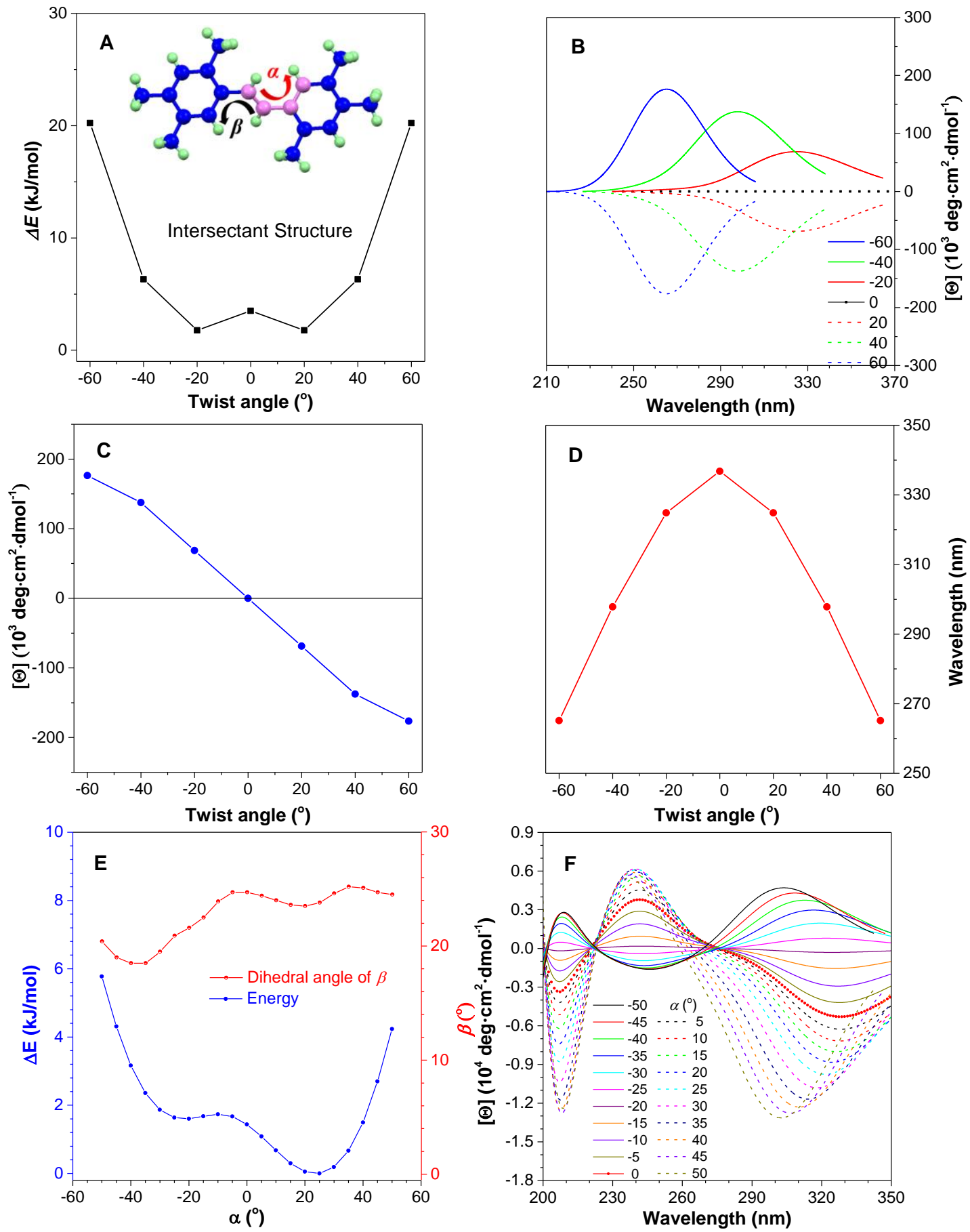

Figure 8. A) Plots single-point energy $(\Delta E)$ versus twist angle of $\alpha$. Intersectant structure of 2,4,5-TMeDPE with $\alpha=\beta$. B) Simulated circular dichroism spectra of 2,4,5-TMe-DPE in intersectant structure with different twist angles. Plots of C) molar ellipticity maximum and D) wavelength maximum versus twist angle of $\alpha$. E) (blue line) Scanning energy curve versus twist angle of $\alpha$, the twist angle of $\beta$ was initially set as $20^{\circ}$; (red line) Plot of twist angle $\beta$ versus $\alpha$. F) Simulated circular dichroism spectra at a different twist angle of $\alpha$, calculated by TD-DFT B3LYP/6-31G(d), Gaussian 09 program. 
According to the chiroptical performances and the corresponding calculation, a primary working mechanism for the AISB was proposed and summarized in Figure 9. When the crystal with parallel conformation was dissolved in its good solvent, there were two pathways for the structural relaxation. 1) C-P to $(R)-\mathrm{C}-\mathrm{I}$ with a rate of $K_{1}$;2) C-P to $(S)$-C-I with a rate of $K_{2}$, C-P: parallel configuration, $\mathrm{C}$-I: intersectant configuration. In the beginning, the proportion of $(R)-\mathrm{C}-\mathrm{I}$ was dominated as $K_{1}>K_{2}$. With the time going on, the intersectant structure trended to packed together due to the hydrophobic environment, then the CD signal from $(R)$-C-I was gradually decreased. Meanwhile, part of the $(R)$-C-I recovered to C-P structures and transformed into $(S)$-CI, which further induced the decrease of $(R)$-C-I signal and the CD signal from $(S)$-C-I gradually dominated. Theoretically, the CD signal would be annihilated with enough standing time. The time-dependent $\mathrm{CD}$ measurement at $f_{\mathrm{w}}=70 \%$ has already proved this hypothesis as the CD signal was disappeared at 550 minutes.

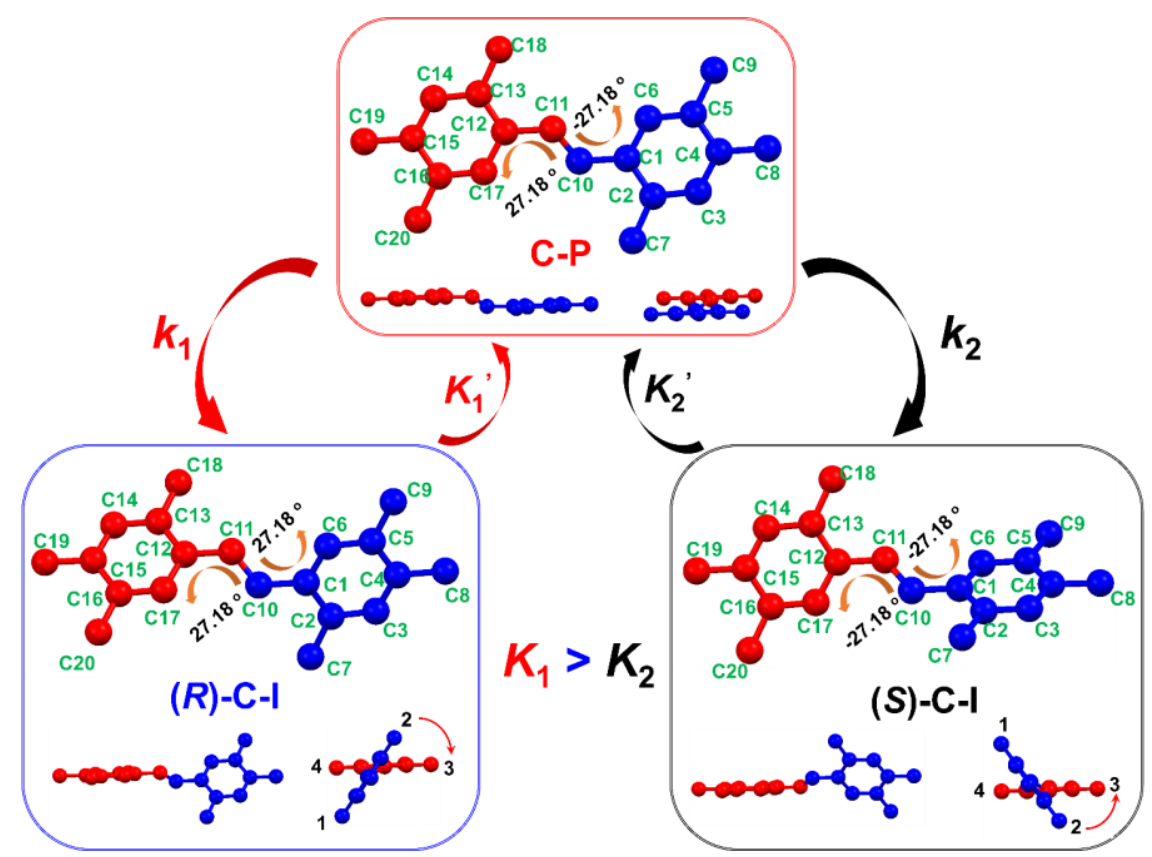

Figure 9. Schematic illustration of the process of kinetic resolution. C-P: parallel configuration, C-I: intersectant configuration, $K_{1}$ : transformation rate from C-P to $(R)$-C-I, $K_{1}{ }^{\prime}$ : transformation rate from $(R)$ C-I to C-P, $K_{2}$ : transformation rate from C-P to $(S)$-C-I, $K_{2}$ : transformation rate from $(S)$-C-I to C-P. 


\section{Conclusion}

A series of achiral diphenylethylene and $p$-terphenyl derivatives were synthesized in this work. However, unexpected chiroptical signals, CD and CPL, were observed in their aggregate state and the emission dissymmetry factor was as high as 0.015 . More interestingly, their CD and CPL properties show a time-dependent effect. For example, no CD signal was achieved for the fresh mixture solution, then negative signal appeared first and its intensity was gradually enhanced with the time going on. At a critical point, the negative signal began to decrease and the positive signal was dominated at last. The theoretical calculation was carried out to prove the existence of two enantiomers in 2,4,5-TMe-DPE, and the experimental CD spectra matched with the simulated ones. Finally, we proposed that the AISB was caused by the difference in transformation rate that the production of $R$-isomer is faster than $S$-isomer. However, the reason for the rate difference is still unknown which needs further exploration.

\section{Acknowledgments}

The authors are grateful for financial support from the National Science Foundation of China (21788102), the Research Grants Council of Hong Kong (16308016, C6009-17G, and AHKUST 605/16), the University Grants Committee of Hong Kong (AoE/P-03/08 and AoE/P- 02/12), the Innovation and Technology Commission (ITC-CNERC14SC01 and ITS/254/17), and the Science and Technology Plan of Shenzhen (JCYJ20160229205601482 and JCY20170818113602462). 


\section{Reference}

1 Zhang, H., Kwok, R. T. K., Lam, J. W. Y. \& Tang, B. Z. SPIE, Liquid Crystals XXII $10735,107350 \mathrm{H}$.

2 Liu, J. et al. What makes efficient circularly polarised luminescence in the condensed phase: aggregation-induced circular dichroism and light emission. Chemical Science 3, 2737, doi:10.1039/c2sc20382k (2012).

3 Song, F. et al. Highly Efficient Circularly Polarized Electroluminescence from Aggregation-Induced Emission Luminogens with Amplified Chirality and Delayed Fluorescence. Advanced Functional Materials 28, 1800051, doi:10.1002/adfm.201800051 (2018).

4 Zhang, H. et al. Axial chiral aggregation-induced emission luminogens with aggregationannihilated circular dichroism effect. Journal of Materials Chemistry C 3, 5162-5166, doi:10.1039/c5tc00629e (2015).

5 Zhang, H. et al. In situ monitoring of molecular aggregation using circular dichroism. Nature communications 9, 4961, doi:10.1038/s41467-018-07299-3 (2018).

6 Haoke, Z. Aggregation-induced emission: mechanistic study, clusteroluminescence and spontaneous resolution Ph.D. thesis, Hong Kong University of Science and Technology, (2018).

7 Zhang, H. et al. Drawing a clear mechanistic picture for the aggregation-induced emission process. Materials Chemistry Frontiers 3, 1143-1150, doi:10.1039/c9qm00156e (2019).

8 Ding, L. et al. Concentration effects in solid-state CD spectra of chiral atropisomeric compounds. New Journal of Chemistry 35, 1781, doi:10.1039/c1nj20185a (2011).

9 Mei, J., Leung, N. L. C., Kwok, R. T. K., Lam, J. W. Y. \& Tang, B. Z. AggregationInduced Emission: Together We Shine, United We Soar! Chemical Reviews 115, 11718 11940, doi:10.1021/acs.chemrev.5b00263 (2015).

10 Sanchez-Carnerero, E. M. et al. Circularly polarized luminescence by visible-light absorption in a chiral O-BODIPY dye: unprecedented design of CPL organic molecules from achiral chromophores. Journal of the American Chemical Society 136, 3346-3349, doi:10.1021/ja412294s (2014). 
11 Shen, Z., Jiang, Y., Wang, T. \& Liu, M. Symmetry Breaking in the Supramolecular Gels of an Achiral Gelator Exclusively Driven by pi-pi Stacking. Journal of the American Chemical Society 137, 16109-16115, doi:10.1021/jacs.5b10496 (2015).

12 Yang, D., Duan, P., Zhang, L. \& Liu, M. Chirality and energy transfer amplified circularly polarized luminescence in composite nanohelix. Nature communications $\mathbf{8}$, 15727, doi:10.1038/ncomms15727 (2017).

13 Xue, S. et al. Unexpected aggregation induced circular dichroism, circular polarized luminescence and helical assembly from achiral hexaphenylsilole (HPS). RSC Advances 7, 24841-24847, doi:10.1039/C7RA02495A (2017). 